\title{
Surfactant Protein D Gene Polymorphism Associated with Severe Respiratory Syncytial Virus Infection
}

\author{
MERI LAHTI, JOHAN LÖFGREN, RIITTA MARTTILA, MARJO RENKO, TUULA KLAAVUNIEMI, \\ RITVA HAATAJA, MIKA RÄMET, AND MIKKO HALLMAN \\ Department of Pediatrics and Biocenter Oulu, University of Oulu, PO Box 5000, FIN-90014 Oulu, \\ Finland [M.L., J.L., T.K., R.H., M.H.], Department of Pediatrics, University of Oulu, PO Box 5000, \\ FIN-90014 Oulu, Finland [Ma.R.], Department of Pediatrics, Seinäjoki Central Hospital, Seinäjoki \\ [R.M.], Laboratory of Developmental Immunology, Department of Pediatrics, Mass General Hospital for \\ Children, 55 Fruit Street, Boston, MA 02114, U.S.A. [Mi.R.]
} \begin{abstract}
ABST
Respiratory syncytial virus (RSV) is the major respiratory
tract pathogen in infancy. Host-related differences in susceptibility to severe RSV infection suggest that genetic factors may play a role. In this study, a candidate-gene approach was used to study whether the surfactant protein D (SP-D) gene polymorphism associates with severe RSV infection. DNA samples from 84 infants hospitalized for the treatment of RSV bronchiolitis and 93 healthy controls were analyzed. The controls were matched with the cases on the basis of sex, hospital district, date of birth $( \pm 2$ wk) and gestational age at birth ( $\pm 2 \mathrm{wk})$. Three biallelic SP-D gene polymorphisms were genotyped. Significant differences were observed in the SP-D allele frequencies for amino acid 11 between the RSV infants and their matched controls. The frequency of the allele coding for Met $11(p=0.033)$ was increased in the severe RSV group. The frequency of the homozygous
\end{abstract}

genotype Met/Met for amino acid 11 was increased in the RSV group relative to the controls, whereas the heterozygous genotype tended to be less frequent among the RSV cases than in the matched controls. Conditional logistic regression analysis was used to study whether the confounders, i.e. smoking and number of children in the family, influence the association between the homozygous SP-D genotype for methionine 11 and the risk of RSV bronchiolitis. The results further confirmed this association $(p=0.028)$. To our knowledge, the present report provides the first evidence of a specific gene associated with susceptibility to severe RSV infection. (Pediatr Res 51: 696-699, 2002)
Abbreviations
RSV, respiratory syncytial virus
SP-D, surfactant protein D

Respiratory syncytial virus (RSV) is a major respiratory tract pathogen in early childhood. In young infants, seasonal epidemics of RSV cause $50 \%$ of the severe instances of bronchiolitis that require hospital treatment and may be fatal. Environmental factors, gender, and socioeconomic status all play a role in RSV infections, but the host-related differences in susceptibility suggest that genetic factors contribute to the risk of contracting this disease (1).

Pulmonary surfactant, a complex mixture of phospholipids and surfactant proteins, lines the alveolar surface of the lung and is essential for normal respiratory function. Surfactant protein D (SP-D) was first described in association with alve-

\footnotetext{
June 28, 2001; October 31, 2001.
}

Correspondence and reprint requests: Mikko Hallman, PhD, Department of Pediatrics and Biocenter Oulu, University of Oulu, PO Box 5000, FIN-90014 Oulu, Finland; e-mail: mikko.hallman@oulu.fi

The study was supported by grants from the Academy of Finland, the Biocenter Oulu, the Sigrid Juselius Foundation and the Foundation for Pediatric Research.

DOI: 10.1023/01.PDR.0000015911.65104.93 olar surfactant. However, this protein does not bind to the surfactant complex and has been found elsewhere in the airways. SP-D is a collagenous C-type lectin mainly assembled as dodecamers consisting of four homotrimeric subunits. SP-D has several immunomodulatory functions, including agglutination of some viruses that cause airway disease (2).

Concentrations of the surfactant components are decreased in viral bronchiolitis $(3,4)$. Studies with a mouse model have shown dysfunction of the surfactant complex in RSV induced pulmonary infections (5). Hickling et al. (6) showed that intranasal administration of recombinant SP-D domain to RSV-infected mice reduced the levels of lung virus by $80 \%$, suggesting that SP-D plays a major role in clearing RSV from the lungs. It is unknown, however, whether SP-D or any other factor involved in innate host defense actually influences susceptibility to RSV bronchiolitis in infants.

Genetic variability may partially explain the differences in individual disease susceptibility. This applies to other surfactant protein genes as well, and they have recently been studied 
as candidates for multifactorial diseases. Associations of certain surfactant protein A (SP-A) and surfactant protein B (SP-B) alleles with the risk of neonatal respiratory distress syndrome have been established (7-9). An association of a SP-D allele with a risk for tuberculosis in a Mexican population was reported recently (10).

Biallelic polymorphisms that alter a single amino acid of the mature protein have been localized in the amino-terminal part (Met11Thr) and the collagenous domain (Ala160Thr) of the SP-D-gene (11). In addition, we have localized a previously unknown biallelic polymorphism Ser270Thr in the carbohydrate recognition domain of SP-D. The aim of the present study was to determine whether these gene variations associate with severe RSV infection.

\section{METHODS}

Subjects. The patients included in the study were admitted into Oulu University Hospital and Seinäjoki Central Hospital during two RSV epidemics between 1998 and 2000. The ethics committees of the participating centers approved the study, and informed consent was obtained from the infants' parents. The controls were selected from the records of newborn infants.

The children with all of the four following criteria were included in the group with severe RSV infection: 1 ) admission into the Children's Hospital in Oulu $(n=72)$ or into the pediatric ward at the Seinäjoki Central Hospital $(n=12)$ with the diagnosis of bronchiolitis, 2) RSV infection confirmed by an antigen test, 3) less than 1 year of age, 4) no other concomitant diseases. The controls were matched with the cases on the basis of sex, hospital district, date of birth $( \pm 2$ $w k)$, and gestational age $( \pm 2 \mathrm{wk})$. The controls had no respiratory infections requiring hospitalizations and a maximum of one ear infection during the infancy. All high-risk premature infants receiving prophylactic Palivizumab monoclonal RSV antibodies or infants with congenital heart disease were excluded from the present study.

Medical data on the cases and controls were collected from the patient records and by means of a questionnaire. The data on neonatal diseases, previous lung diseases, ear infections, allergies, daycare, parental smoking, and the number of children in the family were obtained. More than one control was allowed for each RSV case. Altogether 84 infants with RSV bronchiolitis and 93 controls could be matched according to these criteria. Most of the infants had home daycare during the 1 st year of life ( $98 \%$ of infants with RSV, $96 \%$ of controls), and community-based medical care was available to all at low cost. The characteristics of the RSV infants and their matched controls are presented in Table 1.

SP-D genotyping. Duplicate buccal smears were collected from the cheek mucus with sterile swabs by the parents, or whole blood was obtained. Genomic DNA was extracted from buccal smears using Chelex 100 medium and from whole blood using the Puregene isolation kit (Gentra Systems, Minneapolis, MN, U.S.A.).

Three biallelic polymorphisms at the SP-D codons for the amino acids 11, 160, and 270 in the mature SP-D (12) were genotyped. The Met11Thr genotyping was performed essen-
Table 1. Characteristics of the infants with severe RSV and the matched controls

\begin{tabular}{lcc}
\hline \multicolumn{1}{c}{ Characteristic } & RSV infants $(n=84)$ & Controls $(n=93)^{*}$ \\
\hline Female sex, $n(\%)$ & $30(36 \%)$ & $33(35 \%)$ \\
Male sex, $n(\%)$ & $54(64 \%)$ & $60(65 \%)$ \\
Gestational age at birth & & \\
$\quad($ weeks) & & $39.7 \pm 1.4$ \\
$\quad$ Mean \pm SD & $39.7 \pm 1.7$ & $3598 \pm 506$ \\
Birth weight (g) & & \\
$\quad$ Mean \pm SD & $3572 \pm 562$ & $2.4 \pm 2.2$ \\
Children in family $(n)$ & & $33(35 \%)$ \\
$\quad$ Mean \pm SD & $3.7 \pm 2.5$ & \\
Smoking in family, $\dagger n$ & $33(39 \%)$ & \\
$\quad(\%)$ & &
\end{tabular}

* The controls were matched to the cases for sex, hospital district, date of birth $( \pm 2$ weeks $)$ and gestational age at birth $( \pm 2$ weeks). More than one control was allowed for each RSV patient.

$\dagger$ At least one parent smokes.

tially as described (11), while a PCR-RFLP reaction was used for the Ala160Thr genotyping, in which a 107bp fragment was amplified with the primers SP-D/160F (5'CTCGCAGGCCCTAAGGGAGAG3') and SP-D/160R (5'CTGGACCCAGCCCAGCCCAG3') under standard PCR reaction conditions with a decreasing annealing temperature $\left(67^{\circ} \mathrm{C}-61^{\circ} \mathrm{C}\right)$. The PCR product was digested with MwoI (New England BioLabs, Beverly, MA, U.S.A.), and the resulting fragments, $64 \mathrm{bp}$ and 43bp for Ala and 107bp for Thr, were visualized on a 3\% agarose gel. For the Ser270Thr genotyping, a 115bp fragment was first amplified with the primers SP-D/270F (5'ACGGAGGCACAGCTGCTG3') and SP-D/270R (5'GGAAAGCAGCCTCGTTCT3') at a $58^{\circ} \mathrm{C}$ annealing temperature followed by MnlI (New England BioLabs) digestion and visualization on $3 \%$ agarose. The resulting fragments were $19 \mathrm{bp}, 54 \mathrm{bp}$, and $42 \mathrm{bp}$ for Ser and 19bp and 96bp for Thr. The interassay variability was found to be negligible.

Data Analysis. The observed genotype frequencies were tested for Hardy-Weinberg equilibrium by $\chi^{2}$-analysis. The SP-D allele and genotype frequencies were determined and comparisons of the frequencies were performed by the $\chi^{2}$-test using $2 \times 2$ or $2 \times 3$ contingency tables, respectively. The Woolf logit method was used to determine the odds ratios (ORs) and 95\% confidence intervals (CIs). The software used for the analyses included SPSS for Windows (SPSS Inc., Chicago, IL, U.S.A.) and Arcus Quickstat Biomedical (Longman Software Publishing, Cambridge, UK). Egret statistical software (Cytel Software, Cambridge, MA, U.S.A.) was used for conditional logistic regression analysis.

\section{RESULTS}

Significant differences in the SP-D allele frequencies for amino acid 11 were observed between the 84 infants with severe RSV and the 93 matched controls, the frequency of the allele coding for 11 Met being 0.72 in the RSV group and 0.61 in the control group ( $p=0.033$ ) (Table 2). The frequency of the homozygous genotype Met/Met for amino acid 11 was increased in the RSV group relative to the controls ( 0.55 versus 0.37 ), whereas the heterozygous genotype tended to be less frequent among the RSV cases than in the matched controls 
Table 2. $S P-D$ allele frequencies, odds ratios, and 95\% confidence intervals in the infants with severe RSV and the matched controls

\begin{tabular}{ccccc}
\hline & $\begin{array}{c}\text { RSV infants } \\
(n=84)\end{array}$ & $\begin{array}{c}\text { Controls } \\
(n=93)\end{array}$ & OR $(95 \% \mathrm{CI})$ & $p$-value \\
\hline SP-D allele & & & & \\
$11 \mathrm{Met}$ & 0.72 & 0.61 & $1.63(1.04-2.54)$ & $0.033^{*}$ \\
$11 \mathrm{Thr}$ & 0.28 & 0.39 & $0.62(0.39-0.96)$ & \\
$160 \mathrm{Ala}$ & 0.54 & 0.60 & $0.78(0.51-1.19)$ & 0.251 \\
$160 \mathrm{Thr}$ & 0.46 & 0.40 & $1.28(0.84-1.95)$ & \\
$270 \mathrm{Ser}$ & 0.96 & 0.93 & $2.20(0.82-5.86)$ & 0.108 \\
$270 \mathrm{Thr}$ & 0.04 & 0.08 & $0.46(0.17-1.21)$ & \\
\hline
\end{tabular}

* Statistically significant $p$-values $(p<0.05)$ are indicated by an asterisk. The SP-D allele frequencies were determined and then compared by means of the $\chi^{2}$-test (2-sided) using $2 \times 2$ tables. The Woolf logit method was used to determine the odds ratios (OR) and $95 \%$ confidence intervals $(\mathrm{CI})$.

(0.35 versus 0.49$)$ (Table 3 ). No significant differences were observed in the allele or genotype frequencies for amino acids 160 or 270 . Both groups studied were in Hardy-Weinberg equilibrium.

Conditional logistic regression analysis was used to study whether the confounders, i.e. smoking and number of children in the family, influenced the association between the SP-D genotype and the risk of RSV bronchiolitis. The results of these analyses confirmed the association between the homozygous SP-D genotype $11 \mathrm{Met} / \mathrm{Met}$ and the risk of severe RSV infection $(p=0.028, \mathrm{OR}=2.29,95 \% \mathrm{CI}=1.09-4.81)$.

\section{DISCUSSION}

As a component of innate immunity, SP-D binds and agglutinates some lung pathogens, particularly the common viruses affecting the respiratory tract (2). This may be critical during infancy, when the amounts of placental anti-RSV antibodies are low and the narrow bronchioli are predisposed to inflammatory obstruction. The levels of SP-D are decreased during severe RSV infection (4). In mice, absent expression of SP-D was associated with decreased clearance of respiratory syncytial virus and increased susceptibility to RSV pneumonia (13). Furthermore, administration of SP-D to RSV-infected mice increased the clearance of the virus (6).

Table 3. SP-D genotype frequencies in the infants with severe $R S V$ and the matched controls

\begin{tabular}{cccccc}
\hline & RSV-infants $(n=84)$ & \multicolumn{3}{l}{ Controls $(n=93)$} & $p$-value \\
\hline SP-D genotype & & $(n)$ & & $(n)$ & \\
$11 \mathrm{Met} / \mathrm{Met}$ & 0.55 & 46 & 0.37 & 34 & 0.051 \\
$11 \mathrm{Met} / \mathrm{Thr}$ & 0.35 & 29 & 0.49 & 46 & \\
$11 \mathrm{Thr} / \mathrm{Thr}$ & 0.11 & 9 & 0.14 & 13 & \\
$160 \mathrm{Ala} / \mathrm{Ala}$ & 0.26 & 22 & 0.31 & 29 & 0.369 \\
$160 \mathrm{Ala} / \mathrm{Thr}$ & 0.56 & 47 & 0.58 & 54 & \\
$160 \mathrm{Thr} / \mathrm{Thr}$ & 0.18 & 15 & 0.11 & 10 & \\
$270 \mathrm{Ser} / \mathrm{Ser}$ & 0.93 & 78 & 0.85 & 79 & 0.252 \\
$270 \mathrm{Ser} / \mathrm{Thr}$ & 0.07 & 6 & 0.15 & 14 & \\
$270 \mathrm{Thr} / \mathrm{Thr}$ & 0 & 0 & 0 & 0 & \\
\hline
\end{tabular}

The SP-D genotype frequencies were determined and then compared by means of the $\chi^{2}$-test (2-sided) using $2 \times 3$ tables. Additional comparisons of individual genotypes with $2 \times 2$ tables gave significant results for homozygous genotype $11 \mathrm{Met} / \mathrm{Met}(p=0.015, \mathrm{OR}=2.10,95 \% \mathrm{CI}=1.15-3.84)$ and for heterozygous genotype $11 \mathrm{Met} / \mathrm{Thr}(p=0.045, \mathrm{OR}=0.54,95 \% \mathrm{CI}=$ $0.29-0.99)$.
According to present findings, the polymorphism of the SP-D gene is associated with the risk of severe RSV bronchiolitis in susceptible infants. To our knowledge, the present report provides the first evidence of a specific gene associated with susceptibility to RSV bronchiolitis. Floros et al. (10) recently reported an association of SP-D allele coding for threonine 11 with tuberculosis in Mexican population. Interestingly, in present study from racially homogenous Finnish population, the opposite SP-D allele coding for methionine 11 was associated with severe RSV bronchiolitis in small infants.

Sex, smoking in the family, length of gestation at birth, postnatal age during epidemics and socioeconomic status of the family influence the susceptibility to severe RSV infection. These and other variables had no significant confounding effect on the association between the SP-D gene polymorphism and the disease. In Finland, there are only small differences in the social status of young families and all present cases were hospitalized due to severe symptoms. Eleven percent of the 72 RSV cases in Oulu were treated in pediatric intensive care unit and none among the 12 cases in Seinäjoki required intensive care. Decision to transfer the infant to intensive care is a complex one, and the present material is too small to grade the severity of RSV disease. A large cohort would be required to prospectively analyze the disease characteristics associated with SP-D allele variants. In this study, the controls were matched with the cases on the basis of several important susceptibility factors. In the present racially homogeneous population, community-based medical care is centralized and available at low cost. Neither family size nor smoking in the family explained the differences in SP-D genotype frequencies between the RSV cases and controls, as studied by conditional logistic regression analysis.

We propose that the SP-D Met11Thr polymorphism is a factor explaining the individual disease susceptibility and the severity of RSV bronchiolitis during infancy. The polymorphisms studied here may have functional consequences, because they alter the amino acid composition of the mature protein. The two alternative amino acids, Met and Thr, at position 11 in the mature SP-D molecule have significantly different hydrophobic properties. Amino acid 11 is located near the conserved amino acids Cys15 and Cys20, which are involved in the inter-chain disulfide bonding of the trimeric subunits of the SP-D molecule. These amino-terminal disulfide bridges are required to stabilize the dodecamer structure of SP-D and to maintain its antiviral properties (14). Increasing the extent of multimerization of SP-D increases the antiviral activity of this molecule $(13,15)$. It has been proposed that differences in multimerization could be age or disease related or could result from polymorphisms of SP-D genes that influence assembly (15).

Since SP-D is encoded in a gene cluster that includes other potential candidate genes like surfactant protein A (SP-A) gene, a possibility that the association described here could result from genetic linkage disequilibrium cannot be ruled out. Association studies with multiple comparisons may occasionally produce false positive results. Investigation of other populations would further extend and clarify the association found here. It remains to be studied whether the $11 \mathrm{Met} / \mathrm{Met}$ genotype 
of SP-D has functional consequences like lowered levels in SP-D protein concentrations or deficient antiviral properties against RSV.

The etiology of RSV infection is complex and likely multigenic. The present study provides the first evidence of a gene locus associated with severe RSV infection. Further research is required to assess the complex relationships between genetic and nongenetic factors resulting in the potential for severe RSV disease, the mechanisms involved, and the therapeutic potential of SP-D protein in severe cases of RSV infection.

Acknowledgments. The authors thank Ms. Maarit Hännikäinen for excellent technical assistance.

\section{REFERENCES}

1. Malhotra A, Krilov LR 2000 Influenza and respiratory syncytial virus. Update on infection, management and prevention. Pediatr Clin North Am 47:353-372

2. Crouch E, Hartshorn K, Ofek I 2000 Collectins and pulmonary innate immunity. Immunol Rev 173:52-65

3. Dargaville PA, South M, McDougall PN 1996 Surfactant abnormalities in infants with severe viral bronchiolitis. Arch Dis Child 75:133-136

4. Kerr MH, and Paton JY 1999 Surfactant protein levels in severe respiratory syncytial virus infection. Am J Respir Crit Care Med 159:1115-1118
5. van Schaik SM, Vargas I, Welliver RC, Enhorning G 1997 Surfactant dysfunction develops in $\mathrm{BALB} / \mathrm{c}$ mice infected with respiratory syncytial virus. Pediatr Res 42:169-173

6. Hickling TP, Bright H, Wing K, Gower D, Martin SL, Sim RB, Malhotra R 1999 A recombinant trimeric surfactant protein $\mathrm{D}$ carbohydrate recognition domain inhibits respiratory syncytial virus infection in vitro and in vivo. Eur J Immunol 29:34783484

7. Kala P, Ten Have T, Nielsen H, Dunn M, Floros J 1998 Association of pulmonary surfactant protein A (SP-A) gene and respiratory distress syndrome: interaction with SP-B. Pediatr Res 43:169-177

8. Rämet M, Haataja R, Marttila R, Floros J, Hallman M 2000 Association between the surfactant protein A (SP-A) gene locus and respiratory-distress syndrome in the Finnish population. Am J Hum Genet 66:1569-1579

9. Haataja R, Rämet M, Marttila R, Hallman M 2000 Surfactant proteins A, and B as interactive genetic determinants of neonatal respiratory distress syndrome Hum Mol Gen 9:2751-2760

10. Floros J, Hung-Mo L, Garcia A, Salazar MA, Xiaoxuan G, DiAngelo S, Montano M, Luo J, Pardo A, Selman M 2000 Surfactant protein genetic marker alleles identify a subgroup of tuberculosis in a Mexican population. J Inf Dis 182:1473-1478

11. DiAngelo S, Lin Z, Wang G, Phillips S, Rämet M, Luo J, Floros J 1999 Novel, non-radioactive, simple and multiplex PCR-cRFLP methods for genotyping human SP-A and SP-D marker alleles. Dis Markers 15:269-281

12. Crouch E, Rust K, Veile R, Donis-Keller H, Grosso L 1993 Genomic organization of human surfactant protein D (SP-D). J Biol Chem 268:2976-2983

13. LeVine AM, Whitsett JA 2001 Pulmonary collectins and innate host defense of the lung. Microbes Infect 3:161-166

14. Brown-Augsburger P, Hartshorn K, Chang D, Rust K, Fliszar C, Welgus HG, Crouch EC 1996 Site-directed mutagenesis of Cys-15 and Cys-20 of pulmonary surfactant protein D. J Biol Chem 271:13724-13730

15. White MR, Crouch E, Chang D, Hartshorn KL 2001 Increased antiviral and opsonic activity of a highly multimerized collectin chimera. Biochem and Biophys Res Comm 286:206-213 